\title{
Foro de Arquitectura Tradicional Costeña
}

Edwin Matamoros Chávez

Los días veintitrés y veinticuatro de marzo de 2012 tuvo lugar en Bluefields el Foro de Arquitectura Tradicional Costeña. Este evento fue la culminación de un arduo proceso de trabajo en pro de la puesta en valor de los espacios culturales y el patrimonio arquitectónico de la Costa Caribe. Del documento elaborado para describir la experiencia del encuentro y sus primeros resultados hemos preparado para nuestros lectores esta versión resumida de los antecedentes del evento, las presentaciones, discusiones y acuerdos derivados del Foro.

\section{Antecedentes}

En sus inicios, el Programa Conjunto "Revitalización cultural y desarrollo productivo creativo de la Costa Caribe de Nicaragua" (en adelante, Programa o Programa Conjunto) reconoce que la protección y promoción de la arquitectura tradicional, entendida en su valor como patrimonio de la Costa Caribe, resulta fundamental para la revitalización cultural de los pueblos ${ }^{1}$. La puesta en valor de los espacios culturales repercute no solo en el reforzamiento de las identidades y en la evidencia de la riqueza de la diversidad sino que, además, se concibe como oportunidad de desarrollo desde los principios de cohesión social, convivencia de la ciudadanía y promoción de un turismo cultural.

Sin embargo, en el proceso de ejecución de las actividades del Programa, la misma delimitación del ámbito "arquitectura tradicional" propició varias discusiones acerca de su tipología, la apropiación comunitaria y los valores históricos asociados a las construcciones. Asimismo, en los diagnósticos sobre el estado del patrimonio histórico ${ }^{2}$, promotores culturales y personas consultadas identificaron numerosas infraestructuras, entre edificios y espacios públicos y privados, que consideran representativos de su herencia cultural, y llamaron la atención sobre su vulnerabilidad.

De esta forma se revelaron situaciones inquietantes: por un lado surgió la pregunta ¿cuál es la tipología de la arquitectura tradicional costeña?; por otro lado, ¿cómo proteger esta infraestructura?, pues, una cantidad de los edificios y espacios arquitectónicos identificados se encuentran en franco proceso de deterioro. Con la finalidad de asumir una postura al respecto, que integrara los sectores público, privado, académico y la sociedad civil, nació la idea de crear un espacio para la discusión, el fomento de iniciativas de protección y la toma de decisiones sobre dicha problemática. La dirección del Programa Conjunto denominó "Foro de Arquitectura Tradicional Costeña" a este espacio, concebido para la reflexión y visibilización del tema en la agenda de desarrollo local. Para ello dispuso un equipo facilitador encargado de la organización del evento, al cual encomendó la investigación y consultas sobre el tema ${ }^{3}$, el sitio $\mathrm{web}^{4}$, la toma de fotografías de edificios representativos de arquitectura tradicional costeña y el montaje del evento.

1 La Matriz de Resultados del Programa Conjunto señala: “....habilitar al menos ocho centros comunitarios dedicados a actividades culturales, con diseño acorde a las necesidades, particularidades y arquitectura tradicional de las poblaciones participantes". (Actividad 1.4.1) “...formular propuestas para participar en licitaciones y capacitarlos técnicamente en construcción respetuosa de la arquitectura tradicional" (Actividad 1.5.3)

2 Se realizó un inventario cultural que incluía la infraestructura emblemática para cada uno de los pueblos indigenas y afrodescendientes y para la población costeña en general. Los hallazgos de este mapeo se publican en los cuadernos culturales de la Colección "Identidades y patrimonio cultural" del Programa Conjunto: "Ejecutar el diagnóstico y el mapeo participativo involucrando a las poblaciones participantes y a los actores locales pertinentes y crear la línea de base y directorios". (Actividad 1.1.2 de la Matriz de Resultados)

${ }^{3}$ Las personas consultadas identificaron una relación cercana entre la identidad cultural y la arquitectura tradicional de los pueblos de la Costa y lamentaron la ausencia de iniciativas concretas para preservarlos y promoverlos.

${ }^{4}$ https://sites.google.com/site/arquitecturatradicionalcostena/ 
El foro se estructuró en cuatro grandes momentos: 1) introducción y charlas magistrales; 2) mesas temáticas; 3) plenario, y 4) conformación y primeras actividades de la comisión de trabajo. Para los lectores de Wani, la revista del Caribe nicaragüense, presentamos los puntos medulares de estos apartados.

\section{CHARLAS MAGISTRALES}

La introducción al evento estuvo a cargo de la coordinación del Programa Conjunto, autoridades regionales, de la institucionalidad cultural nacional y de la agencia de Naciones Unidas responsable de la implementación de las actividades que condujeron a la organización del foro. Posteriormente, los arquitectos Accan Sang y Daniel González hicieron sendas disertaciones sobre su experiencia como costeños en el oficio y promoción de la arquitectura costeña.

Las intervenciones magistrales evidenciaron el amplio espectro de actividades edilicias que se desarrollan actualmente y que pueden implementarse en el Caribe de Nicaragua. A continuación presentamos la diversidad temática presentada en el foro:

\section{Patrimonio y Planes de Conservación}

- Algunas directrices básicas para la protección del patrimonio, por José Abraham Yescas (Funcionario INC).

- Hacia una arquitectura de lugar, por Jill Avilés (Consultora UNESCO).

- Patrimonio cultural nicaragüense, Ayzel Palacios García (Funcionaria INC).

\section{Hábitat, construcción y cambio climático}

- Experiencias constructivas de la Iglesia Católica en Waspam, por Floriano Ceferino Vargas (Sacerdote Católico, Parroquia de Waspam).

- Hábitat, construcción y cambio climático, por Luz Marina López (Secretaria de Turismo CRAAN).

- Mano de obra local en restauración y/o rehabilitación, por Bayardo Rueda (Consultor OIT).

\section{Arquitectura e identidad costeña}

- Arquitectura con identidad, por Gundel Tamez (Consultora UNESCO).

\section{Foro de Arquitectura Tradicional Costeña}

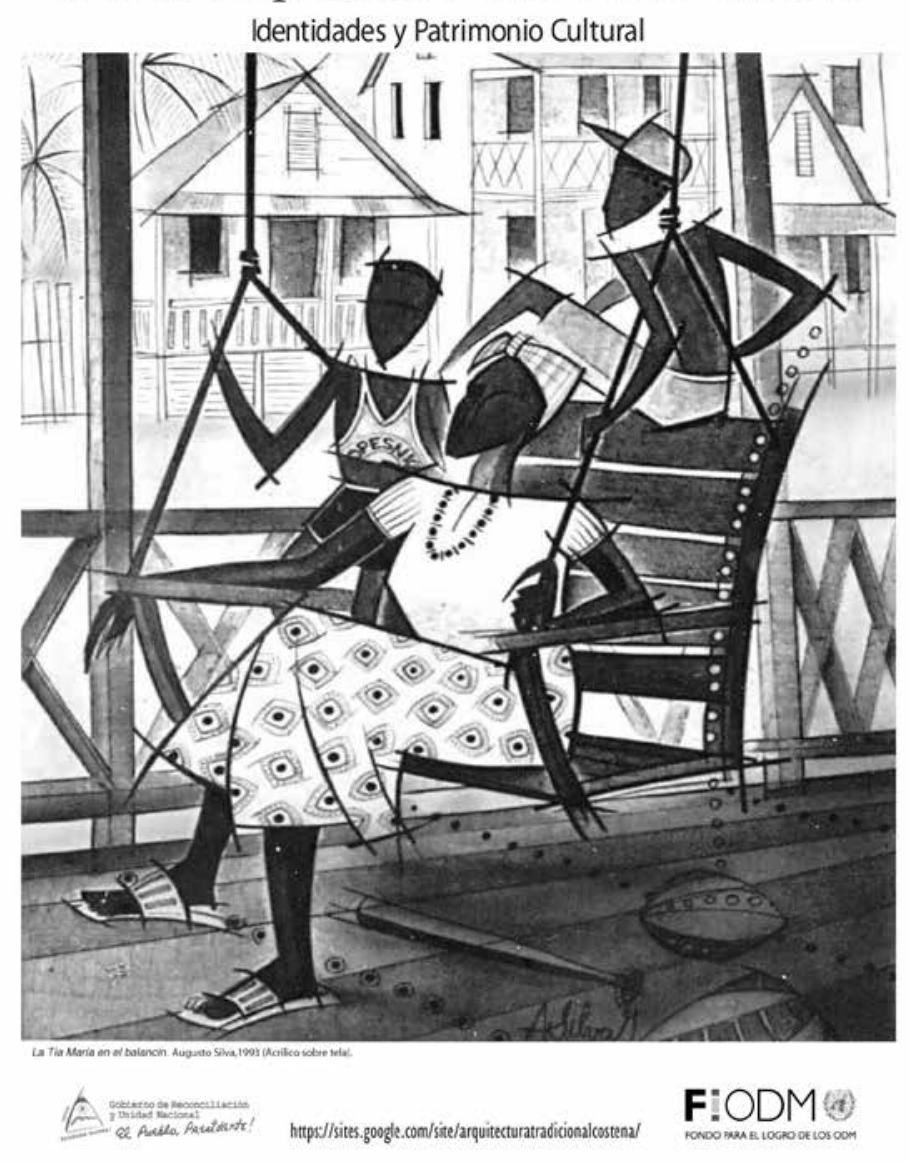

- Centro Integrado de Cultura en Bluefields: manifestando nuestros valores, por Helden Estrada y Yader Chow (Arquitectos, empresa DISENART-Bluefields).

- Mujer costeña en la construcción, por Maritza Chow Zúniga (Arquitecta independiente, Waspam).

\section{MESAS TEMÁTICAS}

Las mesas temáticas fueron iniciativas metodológicas para organizar temas, intereses y canalizar inquietudes. Estas fueron tres: 1) Patrimonio y planes de conservación; 2) Hábitat, construcción y cambio climático, y 3) Arquitectura e identidad costeña. De acuerdo con las hojas de inscripción y de presentación individual de los asistentes, el equipo facilitador motivó a cada uno a integrarse en una mesa particular $^{6}$.

\footnotetext{
5 https://sites.google.com/site/arquitecturatradicionalcostena/3---resultados-del-foro-1/a---mesas-tematicas/arquitectura-con-identidad

${ }^{6}$ El siguiente hipervínculo lleva hacia el sitio web donde éstas se encuentran colocadas: https://sites.google.com/site/ arquitecturatradicionalcostena/3---resultados-del-foro-1/a---mesas-tematicas
} 


\section{Foro de Arquitectura Tradicional Costeña}

Identidades y Patrimonio Cultural

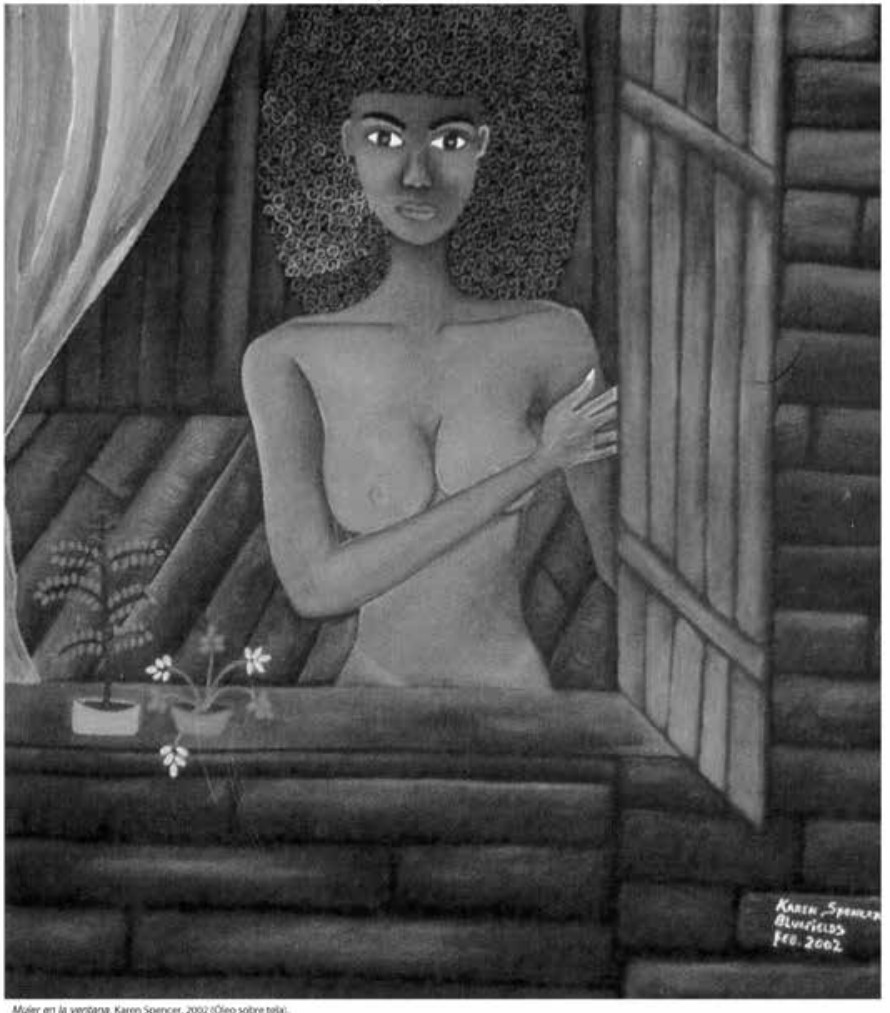

Q2 Axtlo, Pestidents:

hitps://sites.google.com/site/arquitecturatradicionalcostena

$\mathbf{F}: \mathrm{ODM}$

\section{Conclusiones de las mesas temáticas}

a) Falta de apropiación de los valores culturales por parte de la población costeña.

b) Preocupación por la falta de identificación de los bienes culturales en el Caribe.

c) Divulgación de las actividades desarrolladas en relación al mapeo cultural y la valoración de los recursos culturales de las regiones.

d) Poca presencia de técnicos de las alcaldías en el Foro.

e) Cómo llevar estas prácticas culturales a las comunidades de las regiones autónomas.

f) La permanencia/preservación de la esencia de la identidad cultural costeña aún es reconocible.

g) Existe falta de conocimiento, investigación y sensibilización, de la problemática arquitectónica en la región. h) Existe una marcada ausencia del tema de la arquitectura costeña en la educación formal de la población multiétnica.

i) Se necesita de una investigación puntual de cada cultura y arquitectura étnica.

j) Debemos permitir la evolución de la arquitectura moderna, pero incorporando la arquitectura tradicional costeña.

k) No únicamente los arquitectos tienen la responsabilidad social de intervenir en los aspectos arquitectónicos culturales de la población sino, también, la misma sociedad costeña, las comunidades y la entidad gubernamental poseen la capacidad para formular y desarrollar propuestas con pertinencia cultural.

1) El variado uso de los materiales no debe ser la justificación para la producción arquitectónica sin identidad.

m) La falta de uso de materiales tradicionales (madera) se hace sentir debido al agotamiento físico de este recurso y, además, a la falta de técnicas para trabajarlos adecuadamente según los patrones de nuestra arquitectura tradicional.

n) Igual que la cultura evoluciona, la arquitectura también tiene derecho a evolucionar.

o) La arquitectura sigue a la función cultural.

p) El concepto de buen vivir es muy diverso, por lo tanto, la arquitectura debe entender y respetar esos patrones y así partir de esos lineamientos para la creación de los nuevos elementos.

\section{Propuestas acordadas en las mesas temáticas}

a) Implementar la continuación de estudios e investigaciones de cómo cada cultura hace uso de su espacio según sus requerimientos, necesidades y expectativas, para que se brinden respuestas adecuadas, con el objetivo de crear un desarrollo y fortalecimiento de los códigos culturales propios de cada etnia.

b) Llegar a conceptualizar el término de patrimonio entre los costeños.

c) Iniciar las labores de planes de preservación del patrimonio histórico.

d) Fomentar la implementación de normativas (protección, control y regulación) que den paso a un desarrollo integral entre arquitectura y ciudad. 
e) Creación de una red de sociedad civil para la protección del patrimonio cultural (tangible e intangible).

f) Crear los espacios de diálogo para conceptualizar los elementos y características definidos de lo que es la arquitectura costeña, con el objetivo de dar lugar al esparcimiento de todos estos conocimientos a nivel comunal, regional y nacional.

g) El tema de gestión de fondos únicos para la preservación del patrimonio cultural.

h) Establecer políticas y normas de construcción en base a la arquitectura tradicional costeña.

i) Fortalecer los recursos locales, materiales y humanos, en razón de que sean aptos para una construcción que conserve la arquitectura costeña.

j) Concientizar a los gobiernos regionales, territoriales y municipales y a las ONG'S, a preservar la tradición cultural constructiva.

k) Hacer partícipes a las universidades costeñas, con respecto al fomento y desarrollo investigativo de la arquitectura costeña.

1) Mantener este foro en línea para intercambiar experiencias y fomentar nuevas estrategias

m) Presentar planes de conservación de las diferentes culturas.

n) Capacitaciones sobre la identificación e inventario de los recursos culturales (inventario). (INC)

o) Formación de redes culturales comunitarias, para la dinamización de las culturas por medio de los CIC y CCC. (Secretarías de Cultura, INC, Comisiones Comunitarias de Cultura.).

p) Sensibilización y capacitación de los profesionales a cargo de planificación, construcciones e intervenciones en el territorio (INC, Secretarías de Cultura, Unidades Municipales de Cultura).

q) Estudio de las tipologías arquitectónicas junto con las técnicas constructivas y materiales tradicionales costeños (universidades y municipalidades).

r) Elaboración de normativas urbanísticas para las municipalidades.

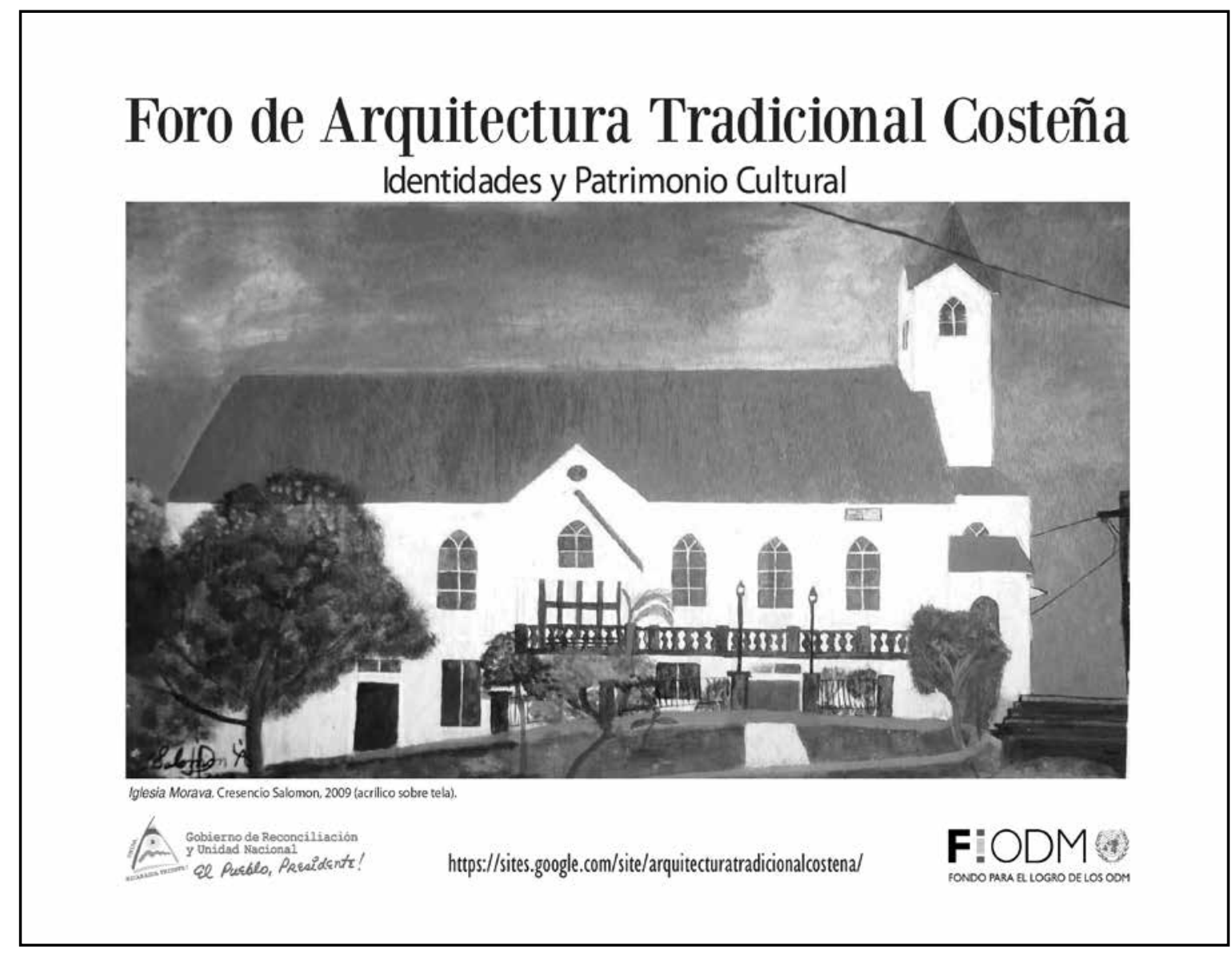




\section{SÍNTESIS DE CONSULTAS}

En el marco de ejecución del Foro se llevó a cabo una consulta con sectores de la población que tienen que ver con la arquitectura y la conservación edilicia en la Región. Estas fueron algunas de las observaciones.

\section{Reflexiones}

- Existen personas que están más enfocadas en resolver problemas habitacionales con recursos accesibles y prácticos para comunidades pobres.

- Responsables de obras de las alcaldías se enfocan en obras públicas, pero en las licitaciones de obras no incorporan criterios que favorezcan la tipología de ATC.

- Propietarios de inmuebles y constructores de obras pequeñas consideran que lo más importante es la calidad y durabilidad de los materiales de construcción.

- Académicos, interesados en varios de los temas anteriormente sugeridos, hacen énfasis en un equilibrio entre estilo, función y costo.

- Empresarios turísticos o urbanísticos miran en el tema una oportunidad para establecer iniciativas conjuntas con actores locales, que sean rentables a ambas partes.

- Algunos consultados asocian el Foro como un momento para abordar soluciones para viviendas populares.

\section{Situación de la arquitectura tradicional costeña}

La existencia y autenticidad de una arquitectura tradicional costeña es un tema a debatir, pues existen expresiones por municipio o territorio, momento, cultura o posición social, que tienen su propia forma de expresión arquitectónica. Existe cierto auge en la construcción de infraestructura habitacional en las ciudades y algunos poblados de la Costa.

En la historia de la humanidad suele ser una práctica el hecho que quienes ostentan mejor posición económica buscan expresarlo a través de la arquitectura. ¿Cómo se expresa esto entre la población costeña? En Bluefields y Bilwi, las entrevistas sugieren que quienes han tenido recursos económico han optado por expresiones de estilo norteamericano, más concretamente, de la región del Mississippi (que es de influencia francesa). Por otro lado, los de menores recursos se conforman con reproducir algunos rasgos de ésta y asegurar la calidad estructural de sus construcciones.

Existe una cantidad de actores trabajando en la construcción de obras de infraestructura habitacional y pública en la costa caribe. Para todos, restauración, conservación y reproducción de arquitectura tradicional costeña constituyen temáticas interesantes a abordar. Sin embargo, pocos están trabajando en estas temáticas. En relación a las obras constructivas que dirigen o en las que participan lamentan no contar con guías o procedimientos para preferir aquellas que tienen contenido de ATC o no.

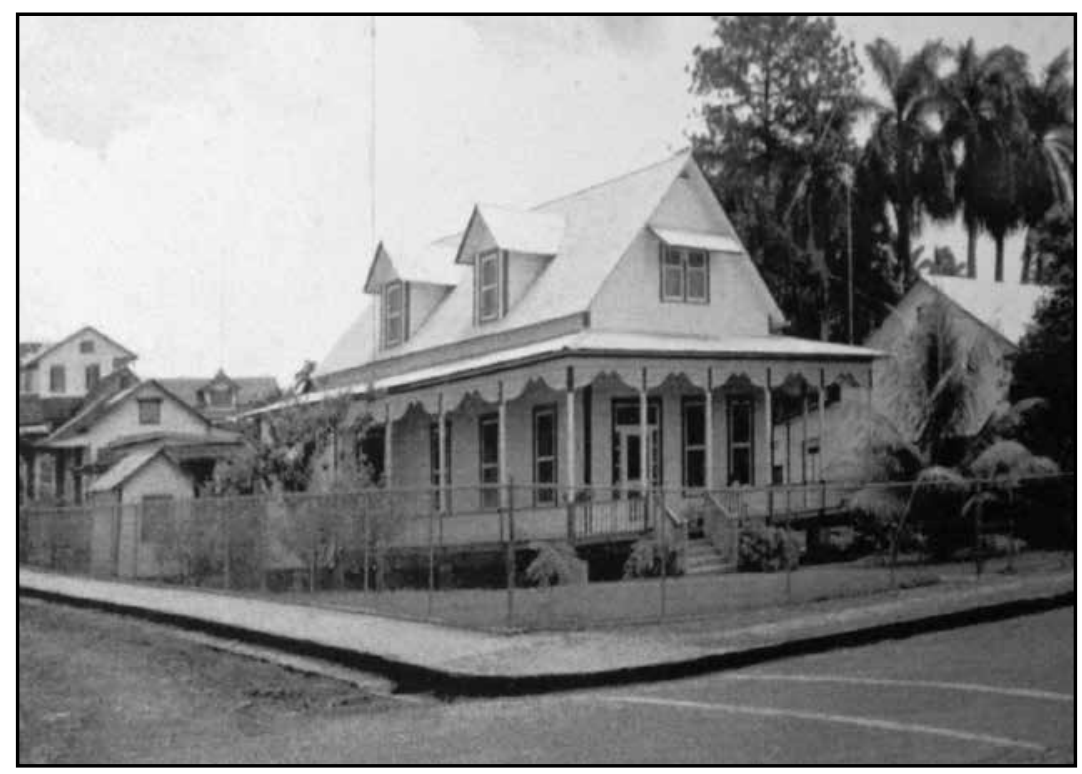

